\title{
An Epidemiologic Study on Hosts and Pathogens of Urinary Tract Infection in Urban Children of Korea (2012-2017)
}

\author{
Yeong Myong Yoo, M.D. \\ Byeong Sub Park, M.D. \\ Shin Young Lee, M.D. \\ Kyu Jung Park, M.D. \\ Hyun Joo Jung, M.D. \\ Ki Soo Pai, M.D, Ph.D. \\ Department of Pediatrics, Ajou \\ University School of Medicine, Suwon, \\ Korea \\ Corresponding author: \\ Ki Soo Pai, M.D., Ph.D. \\ Department of Pediatrics, Ajou University \\ School of Medicine 164 Worldcup-ro, \\ Yeongtong-gu, Suwon 16499, Korea \\ Tel: +82-31-219-5167 \\ Fax:+82-31-219-5169 \\ E-mail:kisoopai@ajou.ac.kr
}

Received: 21 September 2018

Revised: 12 October 2018

Accepted: 18 October 2018

\begin{abstract}
This is an open-access article distributed under the terms of the Creative Commons Attribution Non-Commercial License (http:// creativecommons.org/licenses/by-nc/4.0/) which permits unrestricted non-commercial use, distribution, and reproduction in any medium, provided the original work is properly cited.
\end{abstract}

Purpose: We aimed to determine characteristics of host, causative organisms, and antibiotic susceptibility of bacteria in pediatric patients with UTI living in metropolitan area of Korea.

Methods: Retrospective investigation was done for the causative organisms of UTI in 683 pediatric cases treated at Ajou University Hospital from 2012 to 2017. Patients were classified into Escherichia coli and non-E. coli group, where E. coli group was subdivided into ESBL(+) and ESBL(-) groups based on whether the bacteria could produce extended spectrum beta-lactamase (ESBL). Antibiotic susceptibility of the causative organism was also determined.

Results: A total of 683 UTIs occurred in 550 patients, of which 463 (67.8\%) were first-time infection and 87 (32.2\%) were recurrent ones (2-7 recurrences, 2.52 average), and $64.9 \%$ were male and $35.1 \%$ were female. The most common causative organism was $E$. coli (77.2\%) and ESBL(+) E. coli was found in 126 cases. The susceptibility of $E$. coli to $3 \mathrm{rd}$ or 4 th generation cephalosporin was relatively higher than that to ampicillin or amoxicillin/clavulanic acid. ESBL(+) E. coli showed higher resistance rate to 3rd or 4th generation cephalosporin than ESBL(-) E. coli.

Conclusion: New treatment guideline should be considered due to the incidence of $\operatorname{ESBL}(+) E$. coli increased up to one quarter of UTI cases.

Key words: UTI, Children, Antibiotic resistance, ESBL-producing E. coli

\section{Introduction}

Urinary tract infection (UTI) is one of the most common bacterial infections in children. It is the most common cause of fever without focus in infants younger than two years of age. It accounts for $5-14 \%$ of pediatric emergency room visits ${ }^{1,2)}$. The prognosis of UTI is good in general. However, over $60 \%$ of children with febrile UTI might experience ongoing renal damages resulting in early hypertension, renal insufficiency, or end stage renal failure in adulthood $^{3,4)}$. To prevent renal loss from UTI, it is essential to know the pathophysiology of the disease, including host factors, bacterial factors, and antibiotic resistance ${ }^{5,6)}$. The objective of this study is to determine the recent characteristics of host, pathogens, and antibiotic susceptibility of bacteria in pediatric patients with UTI living in metropolitan area of Korea for successful treatment. 


\section{Materials and methods}

A total 683 pediatric patients aged below 15 years treated for UTI from January 2012 to December 2017 were included in this study. Patients were divided into five age groups (<6 months, 6-12 months, 12-24 months, 24-60, and $\geq 60$ months). Pathogenic strains were grouped as $E$. coli or non-E. coli according to Gram staining, and E. coli group was subdivided into ESBL(+) and ESBL(-) groups depending on whether the bacteria could produce extended spectrum beta-lactamase (ESBL).

UTI was confirmed by body temperature greater than $38^{\circ} \mathrm{C}$ using a tympanic thermometer, urine leukocytes of more than 10 per high power field view on microscopy, and colony count of pathogen at more than $10^{5}$ colonies $/ \mathrm{mL}$ of single organism on urine culture study. When two or more strains were cultured, the cases were excluded from this study $^{7-9)}$.

Urine was collected in a sterilized urine bag or plastic cup after cleaning the urethral orifice area with $2 \%$ boric acid. For microscopic examination, urine samples were centrifuged and Gram stained to observe urinary bacteria and leukocytes. For culture study, $0.001 \mathrm{~mL}$ urine was inoculated into blood agar medium and MacConkey agar medium separately and incubated at $37^{\circ} \mathrm{C}$ for $18-24$ hours. The number of bacterial colonies per $1 \mathrm{~mL}$ was then calculated

${ }^{10)}$. Minimal inhibitory concentration (MIC) to the strain was determined using VITEK2 (Bio-Merieux), a microbial automation device according to the Clinical and Labora tory Standards Institute (CLSI). Bacteria, more than $10^{5}$ colonies $/ \mathrm{mL}$ were considered to be significant as causative agents of UTI.

The antibiotic resistance of $E$. coli strains was analyzed. Resistance rate was expressed as \{ Intermediate+Resistance)/(Susceptible+Intermediate+Resistance)\} for each susceptible, intermediate, and resistant strain to each antibiotic. Bacterial strains with moderate susceptibility in antibiotic sensitivity tests were classified as resistance.

Collected data were analyzed using SPSS 18.0 program. Differences according to gender and age groups were evaluated using Chi-square and t tests. In order to evaluate the difference in incidences among the age groups, goodness of fit was determined by Chi-square test, and cross-tabulation analysis was done to establish significant differences in the incidence of UTI, by gender among the age groups. Statistical significance was considered when $P$ value was less than 0.05 .

\section{Results}

\section{Age and gender distribution of UTI patients}

Among 683 cases, 443 (64.9\%) were male and 240 (35.1 $\%)$ were female. Patients' age less than 6 months accounted for the majority ( $\mathrm{n}=335,49 \%$ ), followed by $6-12$ months ( $\mathrm{n}=192,28 \%), 12-24$ months ( $\mathrm{n}=53,7.8 \%), 24-60$ months $(\mathrm{n}=50,7.4 \%)$ and $\geq 60$ months ( $\mathrm{n}=53,7.8 \%)$. Thus, $77.2 \%$ of childhood UTI developed at the age under 24 months. The incidence of UTI in age $<6$ months group was significantly higher than others $(P<0.001)$, and female incidence was higher only in 24-60 months age group compared to other female age groups $(P<0.001$, Table 1$)$.

\section{Identification of etiologic strains of UTI}

Escherichia coli occurred in most cases ( $\mathrm{n}=527,77.2 \%)$, followed by Klebsiella species ( $\mathrm{n}=73,10.7 \%)$ and Enterobacter species ( $\mathrm{n}=27,4.0 \%$ ). Among 24 patients who showed unusual pathogenic strains of UTI such as Staphylococcus, Enterococcus, and Streptococcus, 10 (42\%) were found to have underlying disease or conditions such as De Lange syndrome, cerebral palsy, corpus callosum agenesis, cerebral infarction, ridden state, and systemic steroid use (Table 2).

\section{Distribution of pathogens according to the age of patients}

Escherichia coli was the most common etiologic strain in all age groups (69.8\% to 79.3\%), and Klebsiella species

Table 1. Age and Gender Distribution of Children with UTI in an Urban Area of Korea (2012-2017)

\begin{tabular}{lrrr}
\hline \multirow{2}{*}{ Age in months } & \multicolumn{2}{c}{ Gender } & \multirow{2}{*}{ Number (\%) } \\
\cline { 2 - 3 } & \multicolumn{1}{c}{ Male } & Female & \\
\hline$<6$ & $254(75.8)$ & $81(24.2)$ & $335(49.0)$ \\
$6-12$ & $118(61.5)$ & $74(38.5)$ & $192(28.1)$ \\
$12-24$ & $34(64.2)$ & $19(35.8)$ & $53(7.8)$ \\
$24-60$ & $12(24.0)$ & $38(76.0)$ & $50(7.3)$ \\
$\geq 60$ & $25(47.2)$ & $28(52.8)$ & $53(7.8)$ \\
Total & $443(64.9)$ & $240(35.1)$ & $683(100.0)$ \\
\hline
\end{tabular}

Abbreviation: UTI, urinary tract infection. 
was the second most common (4.0\% to $17.0 \%)$. Citrobacter was identified $0.9-2.8 \%$ in under 24 months age groups and, Enterobacter $2.6-6.3 \%$ in under 12 months. Proteus mirabilis showed low frequency (1.0-7.7\%) after 6 months of age (Table 3).

\section{Incidence of Escherichia coli according to age and gender of patients}

Incidence of Escherichia coli as the etiology of UTI was the highest (at $84.0 \%$ ) in the age group of 24-60 months. It was not significantly different by gender $(77.0 \%$ in males vs. $77.5 \%$ in females, $P=0.876)$. There was no significant

Table 2. Identification of Pathogenic Strains in Children with UTI (2012-2017)

\begin{tabular}{|c|c|c|}
\hline Gram stain & Strains & $\begin{array}{l}\text { Number of } \\
\text { patients (\%) }\end{array}$ \\
\hline \multirow[t]{19}{*}{ Gram (-) } & Escherichia coli & $527(77.2)$ \\
\hline & Klebsiella species & $73(10.7)$ \\
\hline & K. oxytoca & $10(1.5)$ \\
\hline & K. ozaenae & $2(0.3)$ \\
\hline & K.pneumonia & $61(8.9)$ \\
\hline & Enterobacter species & $27(4.0)$ \\
\hline & E. aerogenes & $17(2.5)$ \\
\hline & E. cloacae & $10(1.5)$ \\
\hline & Citrobacter species & $11(1.6)$ \\
\hline & C. braakii & $1(0.1)$ \\
\hline & C. freundii & $8(1.2)$ \\
\hline & C. koseri & $2(0.3)$ \\
\hline & Proteus mirabilis & $10(1.4)$ \\
\hline & Acinetobacter baumannii & $3(0.4)$ \\
\hline & Morganella morganii & $2(0.3)$ \\
\hline & Pseudomonas aeruginosa & $2(0.3)$ \\
\hline & Raoultella planticola & $2(0.3)$ \\
\hline & Serratia marcescens & $1(0.1)$ \\
\hline & Providencia rettgeri & $1(0.1)$ \\
\hline \multirow[t]{11}{*}{ Gram (+) } & Staphylococcus species & $5(0.8)$ \\
\hline & Coagulase Negative Staphylococcus & $4(0.7)$ \\
\hline & S. aureus & $1(0.1)$ \\
\hline & Enterococcus species & $12(1.8)$ \\
\hline & E. avium & $1(0.1)$ \\
\hline & E. faecalis & $5(0.8)$ \\
\hline & E. faecium & $6(0.9)$ \\
\hline & Streptococcus species & $7(0.9)$ \\
\hline & S. agalactiae & $3(0.4)$ \\
\hline & S.pyogenes & $1(0.1)$ \\
\hline & S. viridans group except S. pneumoniae & $3(0.4)$ \\
\hline
\end{tabular}

Abbreviation: UTI, urinary tract infection. difference among age groups either $(P=0.302)$ (Table 4$).$.

\section{Incidence of $\operatorname{ESBL}(+)$ Escherichia coli by age and gender of patients}

Among a total of 527 E. coli-identified cases, ESBL-producing, ESBL(+) E. coli strains were found in $126(24 \%)$ cases, including 89 (70.6\%) males and 37 (29.4\%) females. Frequencies of $\operatorname{ESBL}(+) E$. coli were not significantly different according to gender or age of patients $(P=0.110$ or $P=$ 0.100 , respectively) (Table 5).

\section{Annual incidence of ESBL(+) Escherichia coli}

A total of $126 \mathrm{ESBL}(+)$ E. coli cases were found, including 5 (4.0\%) in 2012, $22(17.5 \%)$ in 2013, 12 (9.5\%) in 2014, 16 (12.7\%) in 2015, 25 (19.8\%) in 2016, and 46 (36.5\%) in 2017. There was an obvious incremental tendency in the occurrence of $\operatorname{ESBL}(+)$ E. coli cases by year (Fig. 1).

Over a six year-period from January 2012 to December 2017, the occurrence of ESBL $(+)$ E.coli case has increased from $25.0 \%$ in 2012 to $31.9 \%$ in 2017. However, its incidence was not significantly different during these years $(P=0.097$, Table 6)

\section{Antibiotic resistance of general and $\operatorname{ESBL}(+)$ strains of Escherichia coli}

As a result, the resistance rate of ampicillin was 71.7\% and that of amoxicillin/clavulanic acid was $33.6 \%$. Resistance rates against third- and fourth- generation cephalo-

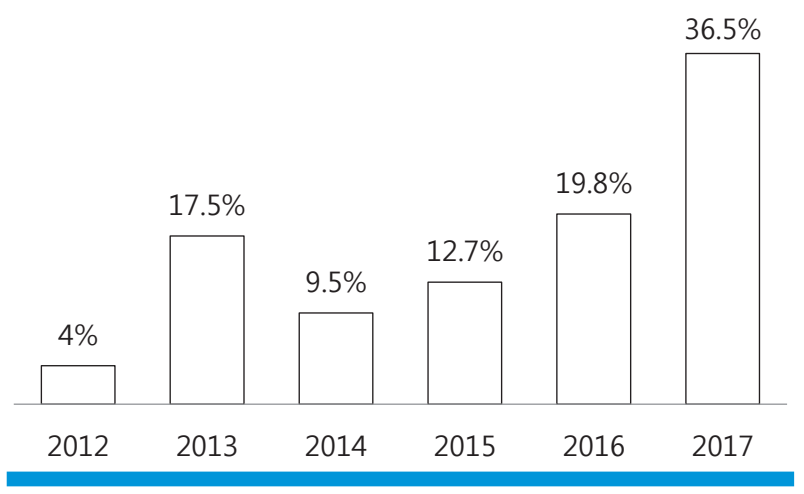

Fig. 1. Annual number of patients with ESBL-producing Escherichia coli in children with UTI living in urban areas of Korea (2012-2017). Extended spectrum beta-lactamase producing, $\mathrm{ESBL}(+)$ E.coli strains were found in 126 patients during the last six years, and the annual report of its occurrence gradually increased from 5 (4.0\%) in 2012, to 46 (36.5\%) in 2017. However, there was no statistical significance in the incidence of $\mathrm{ESBL}(+) E$. coli among total urinary tract infections $(P=0.097)$. 
Table 3. Incidence of Pathogenic Strains of UTI according to Age of Patients (2012-2017)

\begin{tabular}{|c|c|c|c|c|c|c|}
\hline \multirow{2}{*}{ Gram stain } & \multirow{2}{*}{ Pathogens } & \multicolumn{5}{|c|}{ Number of patients (\%) } \\
\hline & & $<6$ mo. & $6-12 \mathrm{mo}$ & $12-24 \mathrm{mo}$. & 24-60mo. & $\geq 60 \mathrm{mo}$. \\
\hline \multirow[t]{9}{*}{ Gram (-) } & Escherichian coli & $265(79.3)$ & $145(75.9)$ & $38(73.1)$ & $42(84.0)$ & $37(69.8)$ \\
\hline & Klebsiella & $29(8.7)$ & $29(15.2)$ & $4(7.7)$ & $2(4.0)$ & $9(17.0)$ \\
\hline & Enterobacter & $21(6.3)$ & $5(2.6)$ & $0(0.0)$ & $1(2.0)$ & $0(0.0)$ \\
\hline & Citrobacter & $3(0.9)$ & $5(2.6)$ & $3(5.8)$ & $0(0.0)$ & $0(0.0)$ \\
\hline & Proteus & $0(0.0)$ & $2(1.0)$ & $4(7.7)$ & $3(6.0)$ & $1(1.9)$ \\
\hline & Acinetobacter & $0(0.0)$ & $0(0.0)$ & $0(0.0)$ & $0(0.0)$ & $3(5.7)$ \\
\hline & Morganella & $0(0.0)$ & $0(0.0)$ & $1(1.9)$ & $0(0.0)$ & $1(1.9)$ \\
\hline & Pseudomonas aeruginosa & $1(0.3)$ & $0(0.0)$ & $0(0.0)$ & $0(0.0)$ & $1(1.9)$ \\
\hline & Serratia marcescens & $1(0.3)$ & $0(0.0)$ & $0(0.0)$ & $0(0.0)$ & $0(0.0)$ \\
\hline \multirow[t]{4}{*}{ Gram (+) } & Enterococcus & $7(2.1)$ & $2(1.0)$ & $1(1.9)$ & $1(2.0)$ & $1(1.9)$ \\
\hline & Staphylococcus & $2(0.6)$ & $1(0.5)$ & $1(1.9)$ & $1(2.0)$ & $0(0.0)$ \\
\hline & Streptococcus & $5(1.5)$ & $2(1.0)$ & $0(0.0)$ & $0(0.0)$ & $0(0.0)$ \\
\hline & Total (100) & $334(49.1)$ & $191(28.1)$ & $52(7.6)$ & $50(7.4)$ & $53(7.8)$ \\
\hline
\end{tabular}

Abbreviation: UTI, urinary tract infection.

Table 4. Incidence of Escherichia coli as the Etiology of UTI according to Age and Gender of Patients (2012-2017)

\begin{tabular}{llrrrr}
\hline & & E. coli & Non-E. coli & $N(\%)$ & $P$-value \\
\hline Sex & Male & $341(77.0)$ & $102(33.0)$ & $443(100.0)$ & 0.876 \\
& Female & $186(77.5)$ & $54(22.5)$ & $240(100.0)$ & \\
& Total & $527(77.2)$ & $156(22.8)$ & $683(100.0)$ & \\
Age in month & $<6$ & $265(79.1)$ & $70(20.9)$ & $335(100.0)$ & 0.302 \\
& $6-12$ & $145(75.5)$ & $47(24.5)$ & $192(100.0)$ & \\
& $12-24$ & $38(71.7)$ & $15(28.3)$ & $53(100.0)$ & \\
$24-60$ & $42(84.0)$ & $8(16.0)$ & $50(100.0)$ & \\
$\geq 60$ & $37(69.8)$ & $16(30.2)$ & $53(100.0)$ & \\
& Total & $527(77.2)$ & $156(22.8)$ & $683(100.0)$ & \\
\end{tabular}

Abbreviation: UTI, urinary tract infection.

sporin were $23-26 \%$. Resistance rate to ertapenem, imipenem, and tigecycline was $0.0 \%$. Antibiotic resistance rates of $\operatorname{ESBL}(+) E$. coli to ampicillin, amoxicillin/clavulanic acid, and third/fourth generation cephalosporin were significantly high; $100.0 \%, 49.2 \%$, and $90.0 \%$, respectively. Their resistance rate to ertapenem, imipenem, and tigecycline was $0.0 \%$, which was the same as the resistance rate of all E. coli strains. Resistance rates to antibiotics other than amikacin were statistically significant between $\operatorname{ESBL}(+)$ and ESBL(-) E. coli $(P<0.05$, Table 7).

\section{Incidence of ESBL(+) pathogens according to recur- rence rate of UTI}

Among 550 patients, ESBL(+) was identified in 146 (26.5 $\%)$. Among these, 126 were E. coli and 20 were Klebsiella
Table 5. Incidence of ESBL-producing Escherichia coli according to Age and Gender of Children with UTI (2012-2017)

\begin{tabular}{llcccc}
\hline & & $\begin{array}{c}\text { ESBL(+) } \\
\text { Ecoli }\end{array}$ & $\begin{array}{c}\text { ESBL(-) } \\
\text { Ecoli }\end{array}$ & N (\%) & $\begin{array}{c}\text { P- } \\
\text { value }\end{array}$ \\
\hline Sex & Male & $89(26.1)$ & $252(73.9)$ & $341(100.0)$ & 0.110 \\
& Female & $37(19.9)$ & $149(80.1)$ & $186(100.0)$ & \\
& Total & $126(23.9)$ & $401(76.1)$ & $527(100.0)$ & \\
Age in month & $<6$ & $60(22.6)$ & $205(77.4)$ & $265(100.0)$ & 0.100 \\
& $6-12$ & $36(24.8)$ & $109(75.2)$ & $145(100.0)$ & \\
& $12-24$ & $14(36.8)$ & $24(63.2)$ & $38(100.0)$ & \\
& $24-60$ & $12(28.6)$ & $30(71.4)$ & $42(100.0)$ & \\
& $\geq 60$ & $4(10.8)$ & $33(89.2)$ & $37(100.0)$ & \\
& Total & $126(100.0)$ & $401(100.0)$ & $527(100.0)$ & \\
\hline
\end{tabular}

Abbreviation: UTI, urinary tract infection; ESBL, extended spectrum betalactamase.

Table 6. Annual Incidence of ESBL-producing Escherichia coli in Children with UTI Living in an Urban Areas of Korea (2012-2017)

\begin{tabular}{lcccccc}
\hline & 2012 & 2013 & 2014 & 2015 & 2016 & 2017 \\
\hline ESBL(+) & 5 & 22 & 12 & 16 & 25 & 46 \\
$\mathrm{n}=126$ & $(25.0)$ & $(26.2)$ & $(21.4)$ & $(18.8)$ & $(18.1)$ & $(31.9)$ \\
ESBL(-) & 15 & 62 & 44 & 69 & 113 & 98 \\
$\mathrm{n}=401$ & $(75.0)$ & $(73.8)$ & $(78.6)$ & $(81.2)$ & $(81.9)$ & $(68.1)$ \\
$\mathrm{N}=527$ & 20 & 84 & 56 & 85 & 138 & 144 \\
$(100.0)$ & $(3.8)$ & $(16.0)$ & $(10.6)$ & $(16.1)$ & $(26.2)$ & $(27.3)$ \\
\hline
\end{tabular}

Abbreviations: UTI, urinary tract infection; ESBL, extended spectrum betalactamase.

species. UTI recurred 220 times in 87 patients; averaging 2.52 and up to 7 times per patient. The recurrence rates of UTI seems to be related with the incidence of $\operatorname{ESBL}(+)$, but there was no significant difference between the frequency 
of $\operatorname{ESBL}(+)$ and relapses of UTI $(P=0.728$, Table 8$)$.

\section{Incidence of VUR according to UTI recurrences}

No significant statistical differences were found in the distribution of the severity of vesico-ureteral reflux (VUR) between recurrent $(\mathrm{n}=87)$ and non-recurrent UTI groups $(\mathrm{n}=463)(P=0.194)$. However, VUR was present significantly higher in recurrent group $(45.9 \%, 34$ out of 74 patients) than in non-recurrent group (33.5\%, 77 out of 230 patients) $(P=$ 0.002 , Table 9). In 87 recurrent UTI patients, the rate of ESBL(+) was not different by the degree of VUR $(P=0.477)$, nor to the presence of VUR $(P=0.618$, Table 10$)$.

\section{Discussion}

Among the 683 pediatric cases diagnosed with UTI at our institute from 2012 to 2017, 64.9\% were male and 35.1\%

Table 7. Comparison of Antibiotic Resistances of Escherichia coli Species according to the Presence of ESBL in Children with UTI (2012-2017)

\begin{tabular}{|c|c|c|c|}
\hline \multirow[b]{2}{*}{ Antibiotics } & \multicolumn{3}{|c|}{ Resistance (\%) } \\
\hline & $\begin{array}{c}\text { General } \\
\text { E.coli strains } \\
(n=527)\end{array}$ & $\begin{array}{c}\text { ESBL }(+) \\
\text { E.coli strain } \\
(n=126)\end{array}$ & $\begin{array}{c}P_{-} \\
\text {value }\end{array}$ \\
\hline AMIKACIN & 0.2 & 0.8 & 0.086 \\
\hline AMOX/CLAVULANIC ACID* & 33.6 & 49.2 & $<0.001$ \\
\hline AMPICILLIN & 71.7 & 100.0 & $<0.001$ \\
\hline AZTREONAM & 23.9 & 99.2 & $<0.001$ \\
\hline CEFAZOLIN & 31.1 & 99.2 & $<0.001$ \\
\hline CEFEPIME & 23.7 & 99.2 & $<0.001$ \\
\hline CEFOTAXIME & 26.9 & 99.2 & $<0.001$ \\
\hline CEFOXITIN & 7.0 & 12.7 & $<0.001$ \\
\hline CEFTAZIDIME & 25.4 & 99.2 & $<0.001$ \\
\hline CIPROFLOXACIN & 23.5 & 38.1 & $<0.001$ \\
\hline ERTAPENEM & 0.0 & 0.0 & NS \\
\hline GENTAMICIN & 24.9 & 44.4 & $<0.001$ \\
\hline IMIPENEM & 0.0 & 0.0 & NS \\
\hline PIPERACILLIN/TAZOBACTAM & 9.9 & 16.7 & $<0.001$ \\
\hline PIPERACILLIN & 69.2 & 100.0 & $<0.001$ \\
\hline TETRACYCLINE & 30.8 & 25.0 & $<0.001$ \\
\hline TIGECYCLINE & 0.0 & 0.0 & $<0.001$ \\
\hline NITROFURANTOIN & 7.7 & 0.0 & 0.004 \\
\hline NORFLOXACIN & 23.1 & 25.0 & $<0.001$ \\
\hline
\end{tabular}

Abbreviations: $\operatorname{ESBL}(+)$, extended spectrum beta-lactamase producing; The general E. coli strains are including $\mathrm{ESBL}(+)$ E.coli group.; NS, no significant difference between $\mathrm{ESBL}(+)$ and general E. coli strains.; * AMOXICILLIN/CLAVULANIC ACID.
Table 8. Incidence of ESBL(+) Pathogens according to the Recurrence Frequency of UTI (2012-2017)

\begin{tabular}{lrrr}
\hline \multirow{2}{*}{$\begin{array}{l}\text { Number of UTI } \\
\text { attacks }\end{array}$} & \multicolumn{3}{c}{ ESBL producibility of pathogens } \\
\cline { 2 - 4 } & ESBL (+) & ESBL (-) & Subtotal \\
\hline 1 & $113(24.4)$ & $350(75.6)$ & $463(84.2)$ \\
2 & $22(36.7)$ & $38(63.3)$ & $60(10.9)$ \\
3 & $6(33.3)$ & $12(66.7)$ & $18(3.3)$ \\
4 & $2(66.7)$ & $1(33.3)$ & $3(0.5)$ \\
5 & $2(66.7)$ & $1(33.3)$ & $3(0.5)$ \\
6 & $1(50.0)$ & $1(50.0)$ & $2(0.4)$ \\
7 & $0(0.0)$ & $1(100.0)$ & $1(0.2)$ \\
Total & $146(26.5)$ & $404(73.5)$ & $550(100.0)$
\end{tabular}

Abbreviations: UTI, urinary tract infection; ESBL, extended spectrum beta-lactamase; Numbers in parentheses are percentages; ESBL(+) strains include both 126 of $E$. coli and 20 of Klebsiella species, respectively.

Table 9. Incidence and Severity of VUR according to the Recurrence of Infections in Children with UTI (2012-2017)

\begin{tabular}{lccc}
\hline \multirow{2}{*}{ VUR Grade } & \multicolumn{2}{c}{ UTI Occurrence } & \multirow{2}{*}{ P-value } \\
\cline { 2 - 3 } & Non-recurrent & Recurrent & \\
\hline No data & 233 & 13 & $0.194^{*}$ \\
0 & 153 & 40 & $0.002^{+}$ \\
1 & 12 & 2 & \\
2 & 24 & 11 & \\
3 & 24 & 11 & \\
4 & 9 & 7 & \\
5 & 8 & 3 & \\
Total & 463 & 87 & \\
\hline
\end{tabular}

Abbreviations: VUR, vesico-ureteral reflux; UTI, urinary tract infection; No data means the corresponding patients had not received VUR study. ${ }^{*} P=0.194$ is for comparison of VUR severity between recurrent and nonrecurrent group.

${ }^{\dagger} P=0.002$ is for comparison of VUR incidence whether UTI recurs or not.

Table 10. Comparison between Severity of VUR and ESBL Producing by Pathogens in 87 Patients with Recurrent UTI

\begin{tabular}{lccc}
\hline \multirow{2}{*}{ VUR Grade } & \multicolumn{2}{c}{ ESBL producing } & \multirow{2}{*}{ P-value } \\
\cline { 2 - 3 } & ESBL $(+)$ & ESBL $(-)$ & \\
\hline No data & 5 & 8 & $0.477^{*}$ \\
0 & 18 & 22 & \\
1 & 0 & 2 & \\
2 & 3 & 8 & \\
3 & 4 & 7 & \\
4 & 3 & 4 & \\
5 & 0 & 3 & \\
Total & 33 & 54 & \\
\hline
\end{tabular}

Abbreviations: VUR, vesico-ureteral reflux; ESBL, extended spectrum beta-lactamase; UTI, urinary tract infection; No data means the corresponding patients had not received VUR study.

${ }^{*} P=0.477$ is for comparison of distribution of VUR severity between ESBL $(+)$ and ESBL(-) group. 
were female. This is similar to previous studies showing a higher prevalence in male children including neonatal period, and higher incidence in females after childhood, except for those aged above 50 years ${ }^{11)}$. High incidence rate of UTI in lower age groups of male might be due to poor penile hygiene during diaper wearing period. Current study indicated the prevalence of UTI in females was lower than that of males in early childhood, but then increased with age: from $24.2 \%$ in age $<6$ months, to $58.2 \%$ in age $>5$ years (Table 1).

Several investigators have reported that pathogens of UTI are mainly enterobacteria, including E. coli and Enterobacteriaceae. Hooton and Stamm have reported that in the United States, E. coli, Staphylococcus, Enterococcus, and other Gram-negative organisms account for $75-90 \%, 5-15 \%$, and $5-10 \%$ of all UTI, respectively ${ }^{12)}$. In the present study, the ratio of $E$. coli was highest at $77.2 \%$, followed by Klebsiella at $10.7 \%$ and Enterobacter at $4.0 \%$, showing that the portion of Gram-negative bacterial infections other than E. coli should be taken into account ${ }^{13,14)}$. UTI is mostly caused by ascending route through colonization and propagation of pathogens of lower gastrointestinal tract to perineum, urethral opening, inside urethra, then finally to the kidney ${ }^{1)}$. Consequentially, most common causative organism of UTI is $E$. coli, while non-E. coli cases account for about $10-35 \%{ }^{15-17)}$. We have a similar result of non- $E$. coli UTI at $23 \%$, whilst a domestic study reported $32.9 \%{ }^{18}$.

We investigated the frequency of ESBL-producing ESBL (+) E. coli strains. UTI caused by ESBL(+) E. coli has been increasing in numbers for the last 6 years. The average prevalence rate of $\operatorname{ESBL}(+) E$. coli was $24.0 \%$ in this study, which is lower than those of foreign reports, at 33-69\%. However, the incidence has increased up to $36.5 \%$ in 2017 , drawing more attention for ESBL(+) E.coli infections. It was also found that 113 out of 463 patients were $\operatorname{ESBL}(+)$ at the time of their first visit, which led to estimate the community prevalence rate of $\operatorname{ESBL}(+)$ uropathogen to be around $24.4 \%$ in children (Table 8 ).

In this study, antibiotics with resistance rates below $10 \%$ were amikacin, cefoxitin, ertapenem, imipenem, piperacillin/tazobactam, tigecycline, and nitrofurantoin. Thirdand fourth- generation cephalosporin such as cefepime (23.7\%) and cefotaxime (26.9\%) showed relatively low resistance rates, whereas $\operatorname{ESBL}(+) E$. coli showed high resistance rate to third- and fourth-generation cephalosporin such as cefepime (99.2\%) and cefotaxime (99.2\%). However, a considerably low resistance rate was displayed against carbapenem series antibiotics such as ertapenem $(0.0 \%)$ and imipenem (0.0\%). For E. coli not producing ESBL, it seems reasonable to consider third- and fourth-generation cephalosporin as the first line antibiotics. For patients with ESBL (+) E. coli cultured or serious clinical conditions, carbapenem series might need to be considered initially.

Nationally, the common regime for UTI treatment is to give empirical antibiotics firstly right after diagnosis, and to discharge the patient or schedule the next visit, if there are apparent clinical improvements for a few days. After completing two weeks of antibiotics administration, the patient will come and receive tests for confirming successful treatment. At times, the patient is informed late that he or she had $\operatorname{ESBL}(+)$ bacterial infection, even though the final follow-up tests are normal: no clinical sign, urine culture negative, and no pyuria. For such cases, we should pay close attention to prevent incomplete treatment or recurrence of UTI.

Throughout the current cases, the recurrence rate of UTI between ESBL(+) and ESBL(-) patients showed no significant difference (Table 8), but greater in patients with vesicoureteral reflux (VUR). Severity of VUR did not affect the recurrence (Table 9).

In Korea, antibiotics are occasionally prescribed for treating simple pediatric febrile illnesses at primary care institutions, and this may lead to increases in antibiotic resistance of bacteria and also to more serious renal damage, due to delayed or incomplete treatment in those misdiagnosed UTI as simple pharyngitis or acute otitis media. It was acknowledged that susceptibility of $E$. coli to third- or fourth- generation cephalosporin was higher than that to ampicillin or amoxicillin/clavulanic acid. Also, $\operatorname{ESBL}(+) E$. coli showed higher resistance rate to third- or fourthgeneration cephalosporin than ESBL(-) E. coli. As a result, empirical choice of antibiotics for UTI treatment may not be safe anymore due to increased frequency of $\operatorname{ESBL}(+) E$. coil up to one quarter of the cases. Therefore, treatment guideline for UTI should be looked into, reflecting the recent findings of antibiotic resistance of pathogens and host characteristics.

Choosing antibiotics for UTI treatment will become more 
difficult as the incidence of $\operatorname{ESBL}(+)$ E.coli is becoming more common. The success rate of primary treatment with empirical antibiotics will be lowered, and, the renal damages will be more serious.

The number of research subjects was much reduced, because this study included single bacterium cultured cases exclusively. In order to obtain more accurate and useful information for UTI treatment, additional researches should be done through extension of research period and expansion of inclusion range of patients.

\section{Patient consent}

This study was approved by the institutional review board (IRB), and the consent was waived due to the nature of the retrospective study [IRB number MED-MDB-18-342].

\section{Conflicts of interest}

No potential conflict of interest relevant to this article was reported.

\section{References}

1. JS E. Nelson Textbook of Pediatrics. 18th ed. Urologic disorders in infants and children. Philadelphia: WB Saunders Co., 2007; 2223-8.

2. Shaikh N, Morone NE, Bost JE, Farrell MH. Prevalence of urinary tract infection in childhood: a meta-analysis. Pediatr Infect Dis J 2008; $27: 302-8$.

3. Hoberman A, Charron M, Hickey RW, Baskin M, Kearney DH, Wald ER. Imaging studies after a first febrile urinary tract infection in young children. N Engl J Med 2003;348:195-202.

4. Hoberman A W.E. Treatment of urinary tract infections. Pediatr
Infect Dis J 1999;18:1020-1.

5. Engel JD, Schaeffer AJ. Evaluation of and antimicrobial therapy for recurrent urinary tract infections in women. Urol Clin North Am 1998;25:685-701.

6. Warren JW, Abrutyn E, Hebel JR, Johnson JR, Schaeffer AJ, Stamm WE. Guidelines for antimicrobial treatment of uncomplicated acute bacterial cystitis and acute pyelonephritis in women. Infectious Diseases Society of America (IDSA). Clin Infect Dis 1999; 29:745-58.

7. Cheng CH, Tsau YK, Lin TY. Effective duration of antimicrobial therapy for the treatment of acute lobar nephronia. Pediatrics 2006;117:84-9.

8. Kass EH. Asymptomatic infections of the urinary tract. 1956. J Urol 2002;167:1016-9.

9. Rubin RH, Shapiro ED, Davis RJ, Stamm WE. Evaluation of new anti-infective drugs for the treatment of urinary tract infection. Infectious Diseases Society of America and the Food and Drug Administration. Clin Infect Dis 1992;15:216-27.

10. Ryu KH, Kim MK, Jeong YB. A Recent Study on the Antimicrobial Sensitivity of the Organisms that Cause Urinary Tract Infection. Korean J Urol 2007;48:603-645.

11. Bahna SL, Torp KH. The sex variable in childhood urinary-tract infection. Acta Paediatr Scand 1975;64:581-6.

12. Hooton TM, Stamm WE. Diagnosis and treatment of uncomplicated urinary tract infection. Infect Dis Clin North Am 1997;11: 551-81.

13. LE N. Epidemiology of urinary infection. Infect Med 2001;18:15362.

14. Stamm WE, Norrby SR. Urinary tract infections: disease panorama and challenges.J Infect Dis 2001;183:S1-4.

15. Ashkenazi S, Even-tov S, Samra Z, Dinari G. Uropathogens of various childhood populations and their antibiotic susceptibility. Pediatr Infect Dis J 1991;10:742-6.

16. Hellerstein S. Urinary tract infections. Old and new concepts. Pediatr Clin North Am 1995;42:1433-57.

17. Hoberman A, Wald ER, Hickey RW, Baskin M, Charron M, Majd M, et al. Oral versus initial intravenous therapy for urinary tract infections in young febrile children. Pediatrics 1999;104:79-86.

18. Jung HJ, Aum JA, Jung SJ, Huh JW. Different characteristic between Escherichea coli and non-Escherichea coli urinary tract infection. Korean J Pediatr 2007;50:457-61. 